\title{
Artikel
}

Christina Thurner*

\section{Bewegte Referenzen. Bei-/Spiele re-/produktiver Abweichung im Tanz}


Abstract: Das Kunstgenre Ballett hat mit seiner oralen beziehungsweise physischen Überlieferungstradition ein ganz eigenes Verhältnis zu Reproduktionen herausgebildet. Dabei sind Wieder- und Neuinszenierungen von Klassikern seit dem 19. Jahrhundert in einer Weise gang und gäbe, die jede Interpretation als eine Art ,Original' ausgibt, zu dem sich wiederum je Referenzsysteme gebildet haben (können). Dieser Beitrag untersucht exemplarisch solche Referenzsysteme im Tanz. Mit Fokus auf die Fortschreibungsgeschichte von Le sacre du printemps soll anhand einer Sacre-Beispielreihe deutlich werden, wie dabei einerseits Bedingungen und Wirkungen von künstlerischer Reproduzierbarkeit (mit-)reflektiert werden und andererseits spielerisch mit Abweichungen umgegangen wird.

Keywords: Original, Urfassung, Reproduktion, Kunstform Tanz, Schwanensee, Le sacre du printemps

* Prof. Dr. Christina Thurner, Institut für Theaterwissenschaft, Universität Bern, Mittelstrasse 43, CH-3012 Bern, email: christina.thurner@itw.unibe.ch

Das Verständnis von ,Original' als ,Urfassung' eines Werks ist hinsichtlich des Genres Ballett problematisch. Die Überlieferungstradition unterscheidet sich da grundlegend von jener in anderen Künsten. Nehmen wir als Beispiel ein berühmtes Ballett: Die meisten Menschen unseres Kulturkreises kennen Schwanensee. Wenn man jedoch genauer nachfragt, hat jede_r vom Schwanensee eine (mehr oder weniger) andere Vorstellung, denkt jede_r dabei an ein anderes Stück, an eine andere Version, eine andere Aufführung, eine andere (Re-)Produktion. ${ }^{1}$ Aber: eine Reproduktion wovon? Diese Frage nach dem ,originären' Werk, auf die man in den Künsten seit der Moderne rasch kommt, stellt sich im Hinblick auf die Kunstform Tanz nicht in derselben Weise. Bleibt man etwa zunächst beim Beispiel Schwanensee, so ist hierbei das ,Original', wenn man denn eines ausmachen will, sogar ziemlich irrelevant.

1 Auf den Begriff der ,Reproduktion' im tanzkulturellen und -historischen Diskurs wird weiter unten noch genauer eingegangen.

\section{1 Übersprungenes Original: Schwanensee}

Als ,Urfassung' oder erste Version nennen verschiedene Lexika und Fachbücher ein Ballett in vier Akten, choreografiert von Julius Wenzel Reisinger zur musikalischen Komposition von Peter Iljitsch Tschaikowski, das unter dem Titel Lebedinoje Osero erstmals 1877 im Bolschoi-Theater in Moskau zu sehen war. ${ }^{2}$ Allerdings wurde dieser "Original Production"3 des Schwanensee-Balletts weder in der Tanzgeschichte beziehungsweise der Tanzgeschichtsschreibung noch in der Tanzpraxis große Bedeutung beigemessen. ${ }^{4}$ Dies mag einerseits daran liegen, dass die zeitgenössische

2 Vgl. beispielsweise Kieser / Schneider 2002, S. 411; Schneider 2016, S. 550; Fanger 2004, S. 29-35.

3 Fanger 2004, S. 29.

4 Vor kurzem erst hat der Choreograf Martin Schläpfer für seine Schwanensee-Produktion an der Oper am Rhein in Düsseldorf, UA im Juni 2018, auf die Version von 1877 zurückgegriffen, was in der Rezeption explizit als Selten- und Besonderheit hervorgehoben wurde; vgl. dazu beispielsweise Weickmann 2018, S. 17. 
Kritik das Stück weitgehend abgelehnt hatte, unter anderem mit dem Argument, Reisinger habe eine allzu schwache Tanzsprache entworfen und die Komplexität von Tschaikowskis musikalisch-psychologischem Drama choreografisch nicht adäquat umgesetzt. ${ }^{5}$ Andererseits ist die Quellenlage zu dieser Version unklar. Es existiert keine überlieferte Aufzeichnung des Balletts und auch das Libretto, das Wladimir Begitschew und Wassili Gelzer zugeschrieben wird, ist offenbar lückenhaft. ${ }^{6}$

Weitaus berühmter und Referenzpunkt für viele Neuchoreographien ist aber nicht die Moskauer, sondern die St. Petersburger Version von 1895. Auf diese Fassung berufen sich bis heute zahlreiche (Re-)Produktionen von Schwanensee. ${ }^{7}$ Dazu schreiben Klaus Kieser und Katja Schneider in Reclams Ballettführer:

Die Popularität von Schwanensee geht [...] nicht auf Wenzel Reisingers Produktion zurück, sondern auf die Inszenierung, die Marius Petipa und Lew Iwanow knapp 20 Jahre später in Sankt Petersburg herausbrachten. Denn diese begründete die bis heute andauernde Aufführungstradition. ${ }^{8}$

5 Vgl. Fanger 2004, S. 30. Vgl. auch Kieser / Schneider 2002, S. 415; innen zufolge bedeutete die Uraufführung für Tschaikowski, der bereits 1875 mit der Komposition begonnen hatte, eine der größten Enttäuschungen seines Lebens. Er fühlte sich und seine Musik offenbar komplett unverstanden, weil Reisinger Teile und Melodien gestrichen und Umstellungen in der Reihenfolge der musikalischen Nummern vorgenommen hatte. Dennoch schreiben Kieser / Schneider 2002, S. 415: "Reisinger [...] hatte eine nach den Maßstäben der Zeit durchschnittlich erfolgreiche Choreografie geschaffen, die sich, mit kleinen Veränderungen, bis 1883 im Repertoire des Bolschoi-Theaters hielt".

6 Vgl. Kieser / Schneider 2002, S. 415. Die Librettisten sollen sich - dies ist immerhin bekannt, vgl. ebd. - auf ältere Quellen bezogen haben, etwa auf das Märchen Der geraubte Schleier aus Johann Karl August Musäus' Sammlung Volksmärchen der Deutschen.

7 Im Bereich des Tanzes wird der Begriff der ,Reproduktion' unterschiedlich verwendet und oft im Zusammenhang mit verwandten Begriffen wie ,Revival', ,Rekonstruktion', ,Remake', ,Restaging' usw. diskutiert. Vgl. dazu Jordan 2000, darin u.a. Hutchinson Guest 2000, S. 65, wobei ich deren enger, sehr spezifischer Definition von ,Reproduktion' nicht folge, sondern vielmehr den Ausführungen von Thomas 2000, S. 125-131, die sich auf Konzepte kultureller Reproduktion, u.a. von Benjamin, beruft; vgl. außerdem Thurner / Wehren 2010.

8 Kieser / Schneider 2002, S. 415.
Indem eine Fortschreibungsgeschichte des Schwanensee-Balletts also den, eigentlichen' Anfang auslässt oder ignoriert, lässt sich die Auffassung von ,Original' als ,ursprünglichem' Anfangspunkt einer Aufführungstradition oder Reproduktionshistorie nicht aufrechterhalten. Das Beispiel Schwanensee, welches hier lediglich stellvertretend als exponiertes Exempel im Bereich Tanz angeführt werden soll, macht deutlich, dass temporal-lineare Bezüge von einem ,Original' hin zu einem ,Fortleben', das heißt von einer ,ersten Version' zu ,Nachfolgeartefakten' wenig sinnvoll bis historisch inkorrekt sind. ${ }^{9}$ Überhaupt ist, wie erwähnt, die Auffassung von ,Original' als ,Urfassung' eines Werks, von dem alle weiteren, die dessen Titel tragen, Reproduktionen oder Kopien sind, im Hinblick auf das Genre Ballett fraglich. Dies einerseits, weil Referenzen aufgrund seiner spezifischen, meist oralen oder genauer: physischen Überlieferungstradition schwer zu ziehen beziehungsweise zu überprüfen sind. Andererseits, weil diese Kunstform unter dem Aspekt der Repertoirepflege ein ganz eigenes Verhältnis zu Reproduktionen herausgebildet hat. Dabei sind Wieder- und Neuinszenierungen von Klassikern im Ballett seit dem 19. Jahrhundert in einer Weise gang und gäbe, die jede Interpretation als eine Art ,Original' ausgibt, zu dem sich wiederum je Referenzsysteme gebildet haben (können). So hat schließlich jede_r berechtigterweise eine (andere) Vorstellung, wenn er oder sie an Schwanensee denkt. Unter den berühmtesten Schwanensee-Versionen zu nennen sind etwa jene von Nicolai Sergejew für das Vic-Wells Ballet (London 1934), die ebenso paradigmatisch in die Tanzgeschichte eingegangen ist, indem sie die Grundlage für die englische Traditionslinie des Werkes bildete, wie John Crankos Stuttgarter Schwanensee (1963), der die Rolle des Siegfried aufwertete, oder Mats Eks psychoanalytisch-moderne Fassung (Umeå 1987) oder Matthew Bournes gender-subversive Version (London 1995) usw. ${ }^{10}$ Das heißt schließlich in Bezug auf das Genre Ballett, dass weniger die Frage interessiert, wie sich eine Version zum ,Original' verhält, als vielmehr jene nach dem Verhältnis einer jeweiligen

9 Vgl. dazu die Kritik an einem solchen zeitlichen Verständnis von ,Original' und dessen ,Fortleben' - mit Referenz auf Benjamin - auch Jäger in diesem Heft.

10 Vgl. auch Kieser / Schneider 2002, S. 418-419. 
Version zu anderen, historischen Versionen. Eine Relationalität der Versionen ist freilich auch im Ballett auszumachen, aber eben nicht linear von einem bestimmten Anfangs- zu einem späteren Fixpunkt, sondern eher netzwerkartig und enthierarchisiert. ${ }^{11}$

Allerdings bedeutet dieser gängige, multiple referentielle Umgang mit Klassikern im Bereich des Balletts nicht, dass im künstlerischen Tanz die völlige Freiheit hinsichtlich Referenzen und Bezügen herrschte. Auch Choreograf_innen halten auf unterschiedliche Weise an der Vorstellung vom ,Original' fest, was bis zu Urheber(rechts)konflikten führt, weil sie beispielsweise ihre ,Original'-Bewegungsabläufe, -Dramaturgien und Bühnensettings kopiert sehen. ${ }^{12}$ Dabei stößt man allerdings auf Probleme: Wie etwa lässt sich zuverlässig bestimmen, ob es sich bei einer Bewegungsfolge um ein ,Original' oder um eine Kopie handelt? Diese Frage wird insbesondere im modernen, postmodernen oder sogenannten zeitgenössischen Tanz virulent, in dem oft nicht (mehr), wie im Ballett, mit normierten, stilisierten, sondern auf der Basis von Alltags-Bewegungen choreografiert wird. Der Berliner Choreograf Christoph Winkler, der diese Problematik 2012 in seinem Stück Dance! Copy! Right? verarbeitet hat, ${ }^{13}$ antwortet denn auch in einem Interview auf die Frage "Kann es ein Urheberrecht an der menschlichen Bewegung geben?": "Tänzer und Choreografen vertreten teilweise extrem konservative Haltungen; es wurde von Diebstahl gesprochen, als hätte sich das Poptheater die letzten zwanzig Jahre lang nicht überall bedient."14

Mit dem Verweis auf das Poptheater referiert Winkler auf eine weitere alternative Auffassung

$11 \mathrm{Vgl}$. zu einer entsprechenden Neubestimmung der Konzepte von ,Original' und ,Kopie' auch Rippl / Stolz in diesem Heft.

12 Vgl. beispielsweise den Streit zwischen Anne Teresa De Keersmaeker und Beyoncé, dazu u.a. Hüster 2011; außerdem Kraut 2016. Genauso interessant wie die schwierige Frage, worin genau die Urheberrechtsverletzung von Beyoncé besteht, ist die Reaktion von De Keersmaeker, die unter dem Titel Re:Rosas! (2013-2014) eine Homepage eingerichtet hat, worauf einerseits die Geschichte des ,kopierten' Stücks, Rosas danst Rosas, dokumentiert wird, und die andererseits allen die Möglichkeit bietet, eigene Versionen hochzuladen, also legal an der Fortschreibung der Produktion mitzuwirken.

13 Vgl. Winkler 2012.

14 Winkler / Kieser 2012. von künstlerischer Re-/Produktion. Die Popkultur hat im Zuge der Postmoderne das ,Original' gewissermaßen abgeschafft und stattdessen intertextuelle und intermediale Verfahren etabliert, die ohne auskommen, indem sie nichtlineare, horizontale, enthierarchisierte Zitier- und Verweisstrukturen erproben. ${ }^{15}$ Im Spannungsfeld von ,Original', ,Zitat', ,Kopie', ,Pastiche', ,Interpretation', ,Adaption', ,Reproduktion' oder ,Aktualisierung' bewegt sich der Bühnentanz zwar ebenfalls auf spielerischem, ${ }^{16}$ wenn auch (nicht nur rechtlich) zuweilen ungesichertem Gelände, aber offenbar anders, als dies herkömmlicherweise etwa der Literatur oder der Bildenden Kunst zugeschrieben wird. ${ }^{17}$

Aufgrund ihres Selbstverständnisses, das auf Konzepten des Performativen, des Hier-und-Jetzt sowie auf einer geradezu topischen Betonung der eigenen Flüchtigkeit beruht und außerdem weitgehend an einer oralen Überlieferungstradition festhält, ${ }^{18}$ schließt die (westliche) Tanzkunst ihre Festschreibung (zumindest weitgehend) aus und entwirft stattdessen eigene Formen der Fortschreibung. Das Ballett reagiert, wie oben ausgeführt, mit der Praxis seiner spezifischen Repertoirepflege, die auf einer beweglich-dynamischen referenziellen Adaption oder einer grundlegenden Neuschreibung basiert. Kreationen werden quasi ohne das Pochen auf ein ,Original' weitergeschrieben, wobei die Handschrift dann freilich auch stark variieren kann. Dagegen produzieren andere künstlerische Tanzgenres wie der moderne, der postmoderne und der zeitgenössische Tanz Stücke, die ebenfalls nicht nachhaltig Originale hinterlassen, weil diese nach einer ersten Kreations- und Aufführungsphase aufgrund ihrer Nicht-Festschreibung auch wieder von der Bühne und damit aus dem kulturellen Gedächtnis verschwinden. ${ }^{19}$

15 Vgl. etwa Baßler u.a. 2016 sowie auch wieder Rippl / Stolz in diesem Heft.

16 Vgl. zum Versuch einer Definition / Abgrenzung der Begriffe auch verschiedene Beiträge in Jordan 2000.

17 Diese Eigenheit teilt die nonverbal-bewegte Tanzkunst wiederum mit anderen performativen Künsten, wobei die Grenzen da heutzutage fließend sind.

$18 \mathrm{Vgl}$. zur oralen Überlieferungstradition und damit zusammenhängend zum Topos der Flüchtigkeit u.a. auch durchaus kritisch - Wehren 2016, S. 15, 97-109.

19 Dieses Phänomen hat - neben dem erwähnten Selbstverständnis der Tanzkunst - verschiedene Gründe, auf die hier nicht genauer eingegangen werden kann. Die entspre- 
Allerdings kennt die Tanzmoderne auch neben der Repertoirepflege des Genres Ballett durchaus bemerkenswert vielfältige referenzielle Fortschreibungsverfahren, die über eine einfache Abfolge von ,Original' zu ,Nachfolgeartefakten' hinausgehen. Darauf möchte ich im Folgenden, ebenfalls wieder an einem Beispiel, eingehen, um aufzuzeigen, wie spielerisch gerade der Umgang mit re-/produktiver Abweichung im Bereich Tanz sein kann. Damit soll nicht zuletzt auch darauf aufmerksam gemacht werden, welche Phänomene in einer interdisziplinären Diskussion zum Verhältnis von Original und Kopie noch stärker in Betracht zu ziehen sind, wenn man dem Thema im Ensemble der Künste gerecht werden möchte.

\section{Dynamische Fortschreibungsgeschichte: Le sacre du printemps ${ }^{20}$}

Innerhalb der Tanzgeschichte reicht mein nächstes Beispiel, Le sacre du printemps, fast an die Berühmtheit von Schwanensee heran. "[V]on allen Choreografien der Moderne wurde dieses Tanzstück wohl am häufigsten in immer wieder neuen Versionen aufgeführt". ${ }^{21}$ Eine Datenbank der Londoner Roehampton University zählt rund 200 Fassungen dieses Tanzstücks zur gleichnamigen musikalischen Komposition von Igor Strawinsky, wobei sie nur die wichtigsten überhaupt erfassen konnte. ${ }^{22}$ Nicht die Zahl der Versionen soll allerdings im Folgenden interessieren, sondern die dynamische Fortschreibungsgeschichte dieses Tanzstücks, genauer: die Verhältnisse der Artefakte zueinander beziehungsweise die Spielarten ihrer Abweichungen, Bezüge und Fortschreibungen. Diese können nämlich wiede-

chende Praxis sowie ihre Problematik wurde in jüngerer Zeit vielfach diskutiert, weil diesen Tanzformen so ihr eigenes Repertoire und ihre Geschichte abhanden kommen. Vgl. dazu u.a. Wehren 2016, S. 42.

20 Die folgenden Ausführungen basieren teilweise auf Recherchen im Rahmen eines Seminars zu Sacre, das ich im Frühjahrssemester 2018 an der Universität Bern angeboten hatte. Ich bedanke mich bei den teilnehmenden Studierenden für deren Anteil an den hier wiedergegebenen Ergebnissen.

21 Brandstetter / Klein 2007, S. 15.

22 Vgl. Jordan / Nicholas 2002. Vgl. auch Jordan 2007b, S. 411; Garafola 2014, S. 165. rum unter diversen Aspekten als unterschiedliche Kategorien, wenn man so möchte, betrachtet werden. Ich schlage für meine exemplarischen Erörterungen folgende drei Begriffe vor, die ich als re-/produktive Kategorien begreifen und je kurz exemplarisch beschreiben sowie aufeinander beziehen werde: 1. ,Original'; 2. Rekonstruktion; 3. Reflexionen.

\section{1 ,Original': Nijinskys Le sacre du printemps (1913)}

Das Choreodrama Le sacre du printemps geht auf eine Kooperation zwischen verschiedenen Kunstformen und Akteuren zurück, ${ }^{23}$ von denen Vaslav Nijinsky (Choreografie), Igor Strawinsky (musikalische Komposition), Nikolai Rjorich (Ausstattung) die drei prominentesten sind. ${ }^{24}$ Es zeigt, ohne narrativ zu sein, die Auserwählung einer Frau, die zur Weihe des Frühlings geopfert wird. Die Pariser Uraufführung durch die Ballets Russes fand am 29. Mai 1913 im Théâtre des Champs-Élysées statt und provozierte einen der größten Theaterskandale der Geschichte. ${ }^{25}$ Die Begründungen für diesen Eklat einerseits und für die mittlerweile über 100-jährige und nach wie vor sehr rege Rezeptionsgeschichte sind vielfältig. ${ }^{26}$ Le sacre du printemps wurde - so die Tanzwissenschaftlerin Gabriele Brandstetter - „in erster Linie als ein ästhetischer Skandal wahrgenommen". ${ }^{27}$ Sie betont hierbei die Bedeutung der Wirkungsgeschichte, die in diesem spezifischen Fall auch die Vorstellung vom ,Original' dieses Stücks prägte und diese (durch die Zeit hindurch) stets dynamisch und beweglich hielt. ${ }^{28}$ Im Gegensatz zu Strawinskys Musik war Nijinskys Choreografie allerdings nach wenigen Aufführungen zunächst von der Bühne verschwunden. Niemand weiß heute mehr, wie dieses Original von 1913 genau aussah, auch wenn zahlreiche Quellen dazu existieren. Die Tanzhistorikerin Lynn Gara-

23 Vgl. zur Gattungsbezeichnung sowie zur Entstehungsgeschichte u.a. Zickgraf 2017.

24 Vgl. auch Kieser / Schneider 2002, S. 399-403.

25 Vgl. dazu u.a. Brandstetter 2017, S. 20-21.

26 Vgl. u.a. Brandstetter 2017, S. 20-35; Garafola 2014, S. 165-179; vgl. auch die Sicht zweier Zeitgenossen: Rivière 2017, S. 313, und Kessler 2005, S. 886.

27 Brandstetter 2017, S. 29. Vgl. dazu auch Woitas 2001, S. 138; außerdem Kieser / Schneider 2002, S. 402.

$28 \mathrm{Vgl}$. Brandstetter 2017, S. 29. 
fola sieht hierin aber genau die Charakteristik der Historie dieses Stücks, indem sie schreibt: „Eben weil es sich um ein verlorenes Ballett handelt, genießt Le sacre du printemps eine konzeptuelle Freiheit, die sowohl die beständige Neuerfindung des Balletts als auch das Fortdauern der mit dem Original verbundenen Vorstellungswelt ermöglicht." ${ }^{29}$ Diese Vorstellungswelt prägen, bei aller Offenheit, dennoch bestimmte Konstanten, durch die sich auch die Fortschreibungen dieses Stücks, das heißt die unterschiedlichen choreografischen Annäherungen seit 1913, charakterisieren lassen. Garafola nennt insbesondere "das Gewaltsame [..., ] das Sexuelle, den Primitivismus, das Erschreckende", 30 kommt allerdings zum Schluss: „Ein Grund - wenn nicht gar der Grund -, weshalb Le sacre du printemps [...] so lebendig bleibt, ist die Tatsache, dass es sich immer wieder als Tanz erneuert." ${ }^{\prime 31}$ Sie erklärt diese stetige Erneuerung mit der "verlockende[n] Möglichkeit, das ursprüngliche Transgressive des Originalballetts immer wieder neu zu erfahren, eine Kunst des Hier und Jetzt an einen Kanon zu knüpfen, der das Einzelwerk transzendiert und vergessene oder halb erinnerte Vorgänger ebenso wie die heute Lebenden einbezieht"; sie folgert: "Noch in seinem hundertsten Jahr bleibt Le sacre du printemps ein , work in progress'."'32

Betont wird hier das Prozesshafte, die Variabilität sowie die Nicht-Fixierbarkeit dieser referentiellen Werkgeschichte. Für deren Beschreibung greift die "klassische Idee des Originalen" - wie Jäger sie nennt -, das heißt eine "chronologische zeitliche und epistemologische Anordnung des Verhältnisses von ,Primärem' und ,Sekundärem', von Vorgängigem und Nachträglichem - sowie die jeweilige Priorisierung des ,Ursprünglichen' vor dem ,Abgeleiteten'", ${ }^{33}$ definitiv zu kurz.

Im Zusammenhang mit dem Thema dieses Bandes interessiert an den zitierten Voten zum Sacre vor allem, wie da zwar von einem ,Origi-

29 Garafola 2014, S. 166; sie begründet dies u.a. mit dem "transgressiven Charakter des Balletts [...] und seiner Zurückweisung überkommener Ballettästhetiken - was alles noch durch den ,Aufruhr' unterstrichen wird, der sich bei der Uraufführung ereignet hat."

30 Garafola 2014, S. 166.

31 Garafola 2014, S. 178; Hervorhebung im Original.

32 Garafola 2014, S. 179.

$33 \mathrm{Vgl}$. Jäger in diesem Heft, S. 14; Hervorhebung im Original. nal' ausgegangen wird, wie aber selbstverständlich anerkannt wird, dass man dieses ,Original' gerade nicht genau kennt, ja dass die Fortschreibung aus diesem Umstand stets neues Potential schöpft und so verschiedene Versionen der Weiterschreibung in immer wieder andere, dynamische Beziehungen zur Leerstelle bzw. zum Phantasma ,Original' setzt.

\subsection{Rekonstruktion: Hodson / Archers Le sacre du printemps (1987)}

Allerdings erfuhr diese Fortschreibungsgeschichte, deren Struktur wiederum nicht-linear, sondern eher netzartig zu verstehen ist, 1987 eine entscheidende Zäsur. Zu diesem Zeitpunkt bezeichneten die britische Choreografin Millicent Hodson und ihr Partner, der Kunsthistoriker Kenneth Archer, ihre Version von Le sacre du printemps mit dem Joffrey Ballet als "Rekonstruktion", was sowohl für ihr Stück als auch für die Nachgeschichte eine entscheidende Bedeutung haben sollte und wirkungsgeschichtlich so weit geht, dass Stephanie Jordan gar die Frage stellt: "The Nijinsky Reconstruction: Birth of an Alternative Tradition?"34, Rekonstruktion' ist freilich ein schwieriger Begriff und erfährt gerade in jüngster Zeit wieder vermehrt Definitionsversuche. Im Tanzbereich existiert seit den 1980/90er Jahren eine teilweise hitzig geführte Debatte, zunächst vor allem im englischsprachigen Raum und nicht zuletzt aus Anlass der viel beachteten, gelobten und auch kritisierten Rekonstruktion des Nijinsky-Stücks Le sacre du printemps durch Hodson und Archer. $^{35}$ Eine konstruktivistisch-kritische Auffassung von ,Rekonstruktion' versteht darunter eine Wiederholung des Kreationsprozesses, also keine identische Wiederholung, sondern eben eine

34 Jordan 2013, S. 231. Weitere Rekonstruktionen durch Hodson / Archer folgten mit dem Paris Opera Ballet (1991); Finnish National Ballet (1994); Companhia Nacional de Bailado, Portugal (1994); Zürcher Ballett (1995); Theatro Municipal Ballet, Rio de Janeiro, Brazil (1996); Rome Opera Ballet (2001); Mariinsky Ballet, St. Petersburg (2003); Birmingham Royal Ballet (2005); Hyogo Performing Arts Centre Ballet, Kobe (2005); Hamburg Ballett (2009); Les Ballets de Monte-Carlo (2009); Filmversion: Mariinsky-Ballet, St. Petersburg (2008); vgl. Hodson / Archer (o. J.).

35 Vgl. z.B. Jordan 2000; Thomas 2004; außerdem Thurner 2016, S. 498-499. 
re-konstruktive, das heißt wieder-herstellende. ${ }^{36}$ So haben denn Hodson und Archer in "jahrelange[r] Detektivarbeit" alle auffindbaren Quellen, die mehr oder weniger direkt auf das ,Original' verwiesen, "zusammengetragen, ausgewertet und wieder zum Leben erweckt. ,Alles war verstreut, es gab keinen Zusammenhang mehr. Wir haben sieben Jahre unseres Lebens voll und ganz der ,Sacre'-Recherche verschrieben', betont Hodson." ${ }^{137}$

Dem großen Vorteil, durch die Rekonstruktion verlorene Werke wieder - zumindest abermals für eine beschränkte Zeit - auf die Bühne holen und dort live oder in einer zeitgemäß-medialen Aufzeichnung erleben zu können, stehen einige Probleme gegenüber, die sich in der Frage zusammenfassen lassen, was man denn da überhaupt genau erlebt. Das ,Original' sicher nicht. Eine Annäherung, etwas nachträglich aus verschiedenen Puzzleteilen Zusammengebasteltes, basierend auf verschiedensten Quellen, die man vom ,Original' noch ,hat'. Von dieser Problematik zeugt exemplarisch der Streit zwischen den Nijinsky-Erben und Hodson / Archer, der 2013 öffentlich wurde anlässlich des 100jährigen Jubiläums der ,Urfassung', zu dessen Feier in Paris auch Hodson / Archers Rekonstruktion gezeigt wurde. ${ }^{38}$ Neben der Frage, wem der Ruhm und auch finanzielle Erlöse für diese Sacre-Re-/Produktion zustehen, interessiert hier vor allem jene nach dem Verhältnis der sogenannten ,Rekonstruktion' zum Phantasma ,Original', denn ",, [t] here was nothing left', lautet das Mantra der Rekonstrukteure, mit dem sie ihren eigenen Copyright-Anspruch untermauern" und ihre eigene Leistung hervorheben. ${ }^{39} \mathrm{Hod}-$ son und Archer halten denn auch in Bezug auf ihre Rekonstruktion fest: „It is a reasonable facsimile.

36 Dabei sind beide Teile des Wortes wichtig: Das ,Wieder' steht für ein ,Nochmals', das nie dasselbe ist wie das ,Ursprüngliche', auf das es sich aber dennoch bezieht. Und das ,Herstellen' meint dezidiert einen Kreationsprozess. Man holt also etwas nicht einfach wieder irgendwo hervor, sondern muss es erarbeiten.

37 Hahn 2013, S. 27.

$38 \mathrm{Vgl}$. zu diesem Urheberrechtskonflikt zwischen Hodson / Archer und der Société des Auteurs et Compositeurs Dramatiques (SACD), die das Copyright und die Rechte der Erben vertritt, das heißt jene der Vaslav and Romola Nijinsky Foundation, der die Nijinsky-Tochter "Tamara vorsitzt", Hahn 2013, S. 27.

39 Hahn 2013, S. 27. Er schreibt weiter: "Was aber heißt: Da war ,nichts'? - darüber gehen die Meinungen auseinander."
[...] Wir wollten dem Original so nahe wie möglich kommen und haben alles über unsere Arbeit publiziert, um eine Debatte zu ermöglichen".40 Und Archer lässt sich zitieren: „'Wir schreiben in unsere Verträge, dass es heißen muss: Rekonstruiert von Millicent Hodson und Kenneth Archer, nach Nijinsky. [...]'"; der Fernsehsender Arte verkaufte allerdings "die ,Sacre'-Rekonstruktion in seiner Live-Übertragung als Nijinskys Choreografie." ${ }^{\prime 41}$ Deshalb wiederum fragt Corinne Honvault von der SACD: "Wer sagt uns, dass die Choreografie den künstlerischen Intentionen Nijinskys entspricht", und weiter: "Sollen doch Hodson und Archer es nicht als Nijinskys, sondern als ihre eigene Arbeit ausgeben! Aber wer hätte diesen ,Sacre' dann genommen?"42

Diese Frage ist eine nach der Art des Verhältnisses von ,Original' und Re-/Produktion. Würde Hodson / Archers, Rekonstruktion' als Re-/Produktion neben anderen betrachtet, ${ }^{43}$ von denen sich viele dadurch auszeichnen, dass sie auf ein früheres Stück, das ,Original', referieren, indem sie dessen Titel und die überlieferte Musik übernehmen und den verloren gegangenen Tanz (wieder beziehungsweise neu) dazu choreografieren, dann würde sie - so zumindest Honvaults ungnädige, aber wohl nicht unberechtigte Annahme - in der Masse der willkürlich mit dem ,Original' verbundenen Nachfolgeartefakte untergegangen sein. Dagegen ist es Hodson und Archer offenbar gelungen, ihren Sacre aus dieser Masse hervorzuheben, gerade indem sie das Verhältnis von ,Original' und ,Rekonstruktion' als ein spezifisches, ein ursächlich-kausal und temporal-linear verlinktes ausgeben und damit dessen Sonderstellung legitimieren. ${ }^{44}$ Die beiden halten fest: „Heute

40 Hodson / Archer zit. nach Hahn 2013, S. 27. Vgl. unter den erwähnten Publikationen etwa Hodson 1980, dies. 1986 / 87, dies. 1996 sowie Archer / Hodson 2000.

41 Hahn 2013, S. 29.

42 Honvault zit. nach Hahn 2013, S. 29.

43 Vgl. zum Verhältnis der Begriffe ,Rekonstruktion', ,Reproduktion' und ,Original' aus tanzwissenschaftlicher Sicht auch Thomas 2000, S. 129.

$44 \mathrm{Vgl}$. dazu die Frage, die Hodson / Archer zit. nach Hahn 2013, S. 27, immerhin stellen: "Ist das Ergebnis authentisch?" Interessant ist allerdings folgender Umstand, den Hahn 2013, S. 29, beschreibt: "Zur Freude der SACD gibt es inzwischen eine zweite Rekonstruktion, die sich von der ersten durchaus unterscheidet." Es ist eine Rekonstruktion, die Dominique Brun unter Einbezug der Nijinsky-Erben erarbeitet hat, die allerdings bis heute kaum jemand kennt. 
wird die Geschichte des Tanzes anders gelehrt, weil unser ,Sacre'-Faksimile existiert. Wir möchten Legenden in die Realität zurückholen." 45 Diese Aussage ist durchaus berechtigt, wenn auch unterschiedlich auslegbar. Das Bild vom ,Original' gibt uns diese ,Rekonstruktion' sicher nicht, auch wenn das Nachleben dieser Sacre-Fassung ein solches Verhältnis suggeriert und sogar Fachleute immer wieder in die Falle tappen, unkommentiert Ausschnitte von Hodson / Archers Rekonstruktion zu zeigen, wenn sie von Nijinskys Sacre sprechen. ${ }^{46}$ Angebracht ist sicherlich ein vorsichtig-kritischer Umgang mit und eine fortgesetzte Reflexion dieses Verhältnisses, wie Jordan sie vorschlägt, indem sie fragt: "So what role within the Sacre tradition did the 1987 Nijinsky reconstruction play?" ${ }^{\prime 4}$

Für den Zusammenhang dieses Beitrags von Bedeutung ist nun folgende Feststellung: Hodson / Archers ,Rekonstruktion' des Sacre, die sich (über umstrittene Legitimationsstrategien) ursächlich auf das ,Original' beruft, ordnete das Verhältnis von ,Original' und dessen Fortleben entscheidend neu. So äußerte denn auch der Philosoph Francis Sparshott bereits 1987 an einer Konferenz anlässlich der Premiere der Sacre-Rekonstruktion die visionäre Vermutung, nachfolgende Choreograf_innen "would now make new versions in relation to the reconstructed ,authentic original', initiating an alternative production tradition." ${ }^{\prime 8}$ Einer Re-/Produktion, die ein kausales, linear-temporales Ver-

Die Gründe dafür mögen vielfältig sein und können hier nicht näher erörtert werden.

45 Hodson / Archer zit. nach Hahn 2013, S. 30. Der Begriff ,Faksimile' taucht in der tanzwissenschaftlichen Rekonstruktionsdebatte meines Wissens sonst nicht auf. Ich denke, er wird von Hodson und Archer hier jedoch vor allem verwendet, um den offenbar problematischen Begriff ,Rekonstruktion' (von einem urheberrechtlich geschützten Werk) zu vermeiden und ihre Produktion als (eigene) Anlehnung, als im etymologischen Wortsinn, ähnlich Gemachtes' zu präsentieren bzw. damit zu legitimieren.

46 Vgl. dazu auch Honvault zit. nach Hahn 2013, S. 27: "Heute wird die Version von Hodson und Archer als die offizielle betrachtet, und das Publikum glaubt mehrheitlich, das Original zu sehen."

47 Jordan 2013, S. 232. Einen Vorteil dieser Debatte sieht Jordan in einem sich seit der Rekonstruktion neu durchzusetzenden historisierenden Zugang zum Stück.

48 Vgl. Sparshott zit. nach Jordan 2013, S. 234. Die Aussage von Sparshott stammt aus dem Panel "The Big Questions", das Sally Banes geleitet hat an der Dance Critics Association and Dance Collection Conference The Rite of hältnis zum ,Original' propagiert, ist es demnach gelungen, einen Paradigmenwechsel in der Fortschreibungsgeschichte auszulösen, indem sie sich quasi als Stellvertreterin für ein verlorenes Original positioniert hat und nun als Referenzpunkt für ein wiederum ganz unterschiedlich sich konstituierendes Bezugssystem figuriert. Für die Fortschreibungsgeschichte des Sacre gilt es deshalb seither zu berücksichtigen, ob und wie sich die verschiedenen Versionen vor und nach $1987 \mathrm{zu}$ dem ,Original' oder aber zu dessen StellvertreterChoreografie verhalten.

\subsection{Reflexionen: Bausch (1975), Le Roy (2007), She She Pop (2014)}

Eine paradigmatische Re-/Produktion des Sacre aus der Vor-Rekonstruktions-Ära ist jene von Pina Bausch, die mit dem deutschsprachigen Titel Frühlingsopfer 1975 innerhalb eines dreiteiligen Abends im Opernhaus Wuppertal erstmals aufgeführt wurde und sich bis heute im Repertoire des Wuppertaler Tanztheaters befindet. ${ }^{49}$ Das Stück wird dem Frühwerk von Bausch zugeordnet, ${ }^{50}$ in dem diese sich einerseits (noch) choreografisch integral mit bestehenden Musikstücken auseinandersetzte (im Fall von Frühlingsopfer mit dem Strawinsky-Sacre in der Interpretation von Pierre Boulez $)^{51}$ und andererseits gesellschaftskritische Themen (allen voran Geschlechter- und Rollenverhältnisse) behandelte. Bauschs Frühlingsopfer kann folgendermaßen beschrieben werden. ${ }^{52} \mathrm{Zu}$ Beginn des Stücks liegt eine Frau bäuchlings auf einem roten Tuch am Boden, der mit Torf belegt ist.

Spring at Seventy-Five in der New York Public Library for the Performing Arts, Lincoln Center, 1987.

49 Bausch 1975. Vgl. zur Bedeutung von Bauschs Frühlingsopfer in der Werk- und in der Tanzgeschichte auch Brandstetter / Klein 2007, S. 15, wobei in Bezug auf den Titel des Stücks interessant ist, dass in der Erstausgabe des Bandes Bauschs Stück noch mit "Le sacre du printemps" betitelt ist, während in der überarbeiteten Neuauflage von 2015 bereits im Untertitel des Buches "Le sacre du printemps / Das Frühlingsopfer" steht. Auf die Übersetzung / Übersetzbarkeit des Titels als - durchaus relevanter - Aspekt der Fortschreibungsgeschichte kann hier nicht näher eingegangen werden.

50 Vgl. zum Frühwerk von Bausch u.a. Schlicher 1987, S. 108-149.

51 Vgl. dazu Jordan 2007a, u.a. S. 161.

52 Vgl. zum Folgenden auch Thurner 2007. 


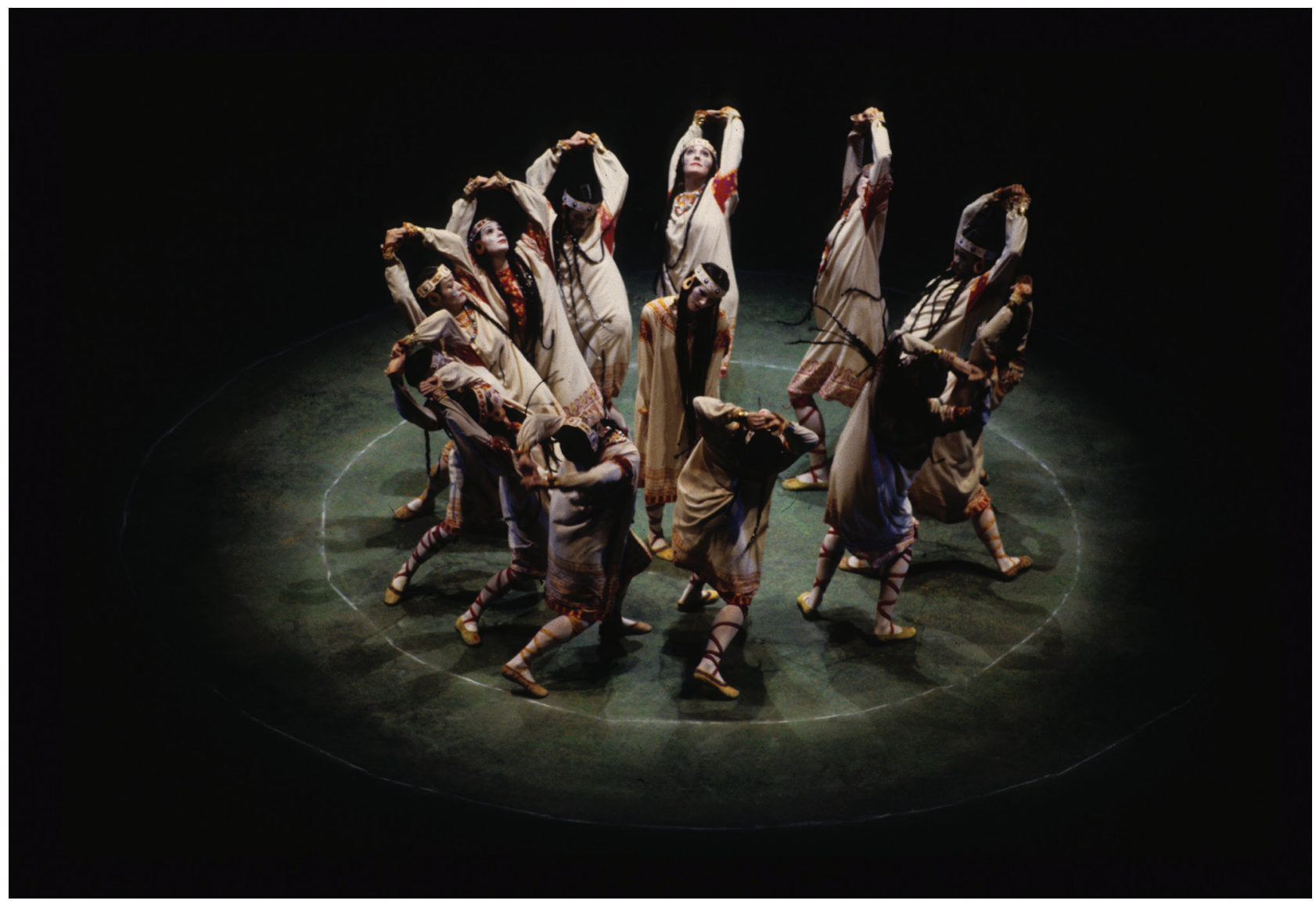

Abb. 1. Le sacre du printemps. Rekonstruktion von Millicent Hodson / Kenneth Archer / Joffrey Ballet. Foto: Herbert Migdoll mit freundlicher Genehmigung des Joffrey Ballet

Es macht den Anschein, als ströme Blut aus ihrem Oberkörper. Nach und nach treten weitere Frauen auf und tanzen einzeln oder in verschiedenen Raum- und Gruppenformationen. Dabei zieht sich eine von den Armen ausgehende, autoaggressive Bewegung leitmotivisch durch die Choreografie und variiert in ihrer Dynamik analog zur Musik. Die erst später auftretenden Männer nehmen mit kräftigen Bewegungen und mit Sprüngen das Territorium ein. Inzwischen wird das rote Tuch, das sich am Ende als Kleid für die Auserwählte erweist, als Zeichen für die bevorstehende Opferung von Frau zu Frau gereicht. Die mit dem Tuch in Berührung gekommenen Frauen winden sich in konvulsiven Bewegungen. Das Opfer (hier im doppelten Wortsinn von Person und Handlung) ist in Pina Bauschs Version des Sacre eine weibliche Angelegenheit der Verausgabung. In einer Szene gegen Ende bilden die Tänzerinnen schließlich einen engen Kreis, aus dem immer wieder eine ausschert, um das Tuch einem Mann hinzuhalten, wieder umzudrehen und in den Kreis der
Frauen zurückzukehren. Das Opfer der Frau wird dem Mann also angeboten, hingehalten, es wird nicht mit inm geteilt, sondern ihm mitgeteilt. Es findet kein Tauschakt statt. Interessant ist, dass dieser Kreis der Opfererwählung im Gegensatz zu Nijinskys Version leer ist. Niemand steht als Opfer in der Mitte des Kreises. Das potentielle Opfer liegt im einzelnen Frauenkörper, der geopfert wird. Die Opferung trifft dabei nicht eine vorbestimmte Auserwählte, sondern ist eine soziale Aushandlung.

Auf diese Sequenz der Aushandlung folgt bei Bausch, analog zur plötzlich einsetzenden Vehemenz der Musik, die letzte Verausgabung. Die Frauen drehen sich um ihre eigene Achse, springen heftig gegen die Männer an. Eine Frau, nun im roten Kleid, und ein Mann stehen in dieser bewegten Dynamik still: das Opfer und jener, der sie zur Opferung führen wird oder ihr das Opfer, die Geste der Verausgabung, abnehmen wird. Die Opferung ist somit potentiell vervielfältigt und auswechselbar, jede kann die Rolle austragen, weil sie sie 
potentiell in sich trägt. Die eigentliche Stätte der Opferung liegt, so lese ich Bauschs Stück auch im Gender-Kontext der 1970er Jahre, im Innern einer jeden Frau. Diejenige, die am Schluss exponiert zu Tode kommt, wiederholt dabei nur die autoaggressiven Bewegungen, die vorher alle ihre Mittänzerinnen auch ausgeführt haben. Dass sie es also ist, die am Schluss bäuchlings auf der Erde liegt, ist kontingent, es hätte genauso eine andere treffen können. Auch die erlösende Wirkung wird schließlich nicht eingelöst, weil das Opfer kontingent ist, die Mechanismen der Opferung unter anderem durch Wiederholungen ausgestellt und entlarvt werden und auch das Publikum durch die halbkreisförmige Anordnung der Tanzenden am Ende in den aggressiven Zusammenschluss mit einbezogen wird.

Diese Lesart macht deutlich, dass Bauschs Stück das ,Original', von dem die Choreografin 1975 lediglich die Partitur, musikalische Aufnahmen, allenfalls Beschreibungen und vielleicht frühere Re-/Produktionen kennt, als eine Art ,Folie' behandelt. Auf Nijinskys Version beziehungsweise auf die damit verbundene, von Garafola so genannte "Vorstellungswelt" ${ }^{\prime 2}$ referiert sie einerseits semantisch sowie atmosphärisch, indem sie deren (vage) Attribute wie gewaltig, energetisch und erschreckend aufnimmt, und andererseits formal durch ihre Reflexion der Kreisformationen und die genaue Umsetzung der (von der Musik geprägten) komplexen Rhythmisierung und (leitmotivischen) Wiederholungen. Ihre Choreografie ist aber weder der Versuch einer mimetischen Re-/Produktion des ,Originals', noch nähert sie sich ihm überhaupt ästhetisch an, sie begibt sich vielmehr zwar in dessen Vorstellungswelt, reflektiert aber diese, indem sie zeitgemäße Anliegen darüberprojiziert. Dabei ist die Stelle, auf die sich Bauschs Referenz bezieht, genauso leer beziehungsweise imaginär wie jene inmitten ihres Kreises der potenziellen Opfer.

Man könnte noch zahlreiche weitere Beispiele für solche referenziellen Reflexionen beschreiben, die sich in ihrer jeweiligen Bezugnahme dezidiert von mimetischen Reproduktionen und damit von Rekonstruktionen abheben. ${ }^{54}$ Auf zwei in ihrer Art

53 Garafola 2014, S. 166.

$54 \mathrm{Vgl}$. zu den dezidiert referentiellen Reflexionen, auch zu verstehen als produktive Kulturtechnik der Mittelbarkeit, den Beitrag von Peter Schneemann in diesem Heft. der Relationalität signifikante möchte ich im Folgenden exemplarisch noch kurz eingehen. Dieses reflexive Spiel mit Vorstellungswelt, Leerstelle und Abweichung auf die Spitze treibt Xavier Le Roy. Der lange Zeit in Berlin ansässige Franzose hat 2007 ein mit Le sacre du printemps betiteltes Stück kreiert, ${ }^{55}$ in dem weder ein Opfer noch Tanz im herkömmlichen Sinn zu sehen sind. ${ }^{56}$ Die Musik, die während des Stücks zu hören ist, ist Strawinskys Sacre in einer Aufnahme der Berliner Philharmoniker, dirigiert von Simon Rattle. Zu dieser Musik dirigiert Le Roy auf der Bühne, wobei er so tut, als bilde das Publikum das Orchester. ${ }^{57}$ Unter den Sitzen der Zuschauenden sind Lautsprecher versteckt, die - gemäß der Anordnung eines Symphonieorchesters - den Ton der entsprechenden Instrumente wiedergeben. ${ }^{58}$ Dadurch bekommt der einzelne Besucher tatsächlich das Gefühl, Teil des Orchesters und damit Adressat von Le Roys Gesten beziehungsweise an der Produktion der musikalischen Aufführung beteiligt zu sein. Diese wiederum referiert auf ein Projekt, das der Theaterwissenschaftler Clemens Risi folgendermaßen beschreibt:

Als eines der erfolgreichsten und gleichzeitig meist dis-
kutierten Beispiele kultureller Bildung im Bereich des
Tanztheaters und der Musik gilt das vor allem durch
den Kinofilm berühmt gewordene Projekt Rhythm Is It,
ein im Jahr 2003 gemeinsam von den Berliner Philhar-
monikern, ihrem Chefdirigenten Sir Simon Rattle und
dem Choreographen Royston Maldoom durchgeführ-
tes Tanzprojekt zur Musik von Strawinskys Le Sacre
du Printemps für und mit SchülerInnen verschiedener
Berliner Schulen, insbesondere auch aus Problembe-
zirken. ${ }^{59}$

Ohne hier auf all diese Referenzen und insbesondere auf dieses - meines Erachtens zu Recht umstrittene - Community-Dance-Projekt genauer eingehen zu können, möchte ich am Beispiel von Le Roys Sacre einerseits hervorheben, welche Komplexität das Bezugssystem dieses Stücks mittlerweile erlangt hat. Eine Komplexität, die netzartig verwoben in ganz verschiedene

55 Vgl. Le Roy 2007a.

562018 hat Le Roy eine zweite, divergierende Version kreiert, in der mehrere Tänzer_innen auf der Bühne agieren. Auf diese Version wird hier jedoch nicht eingegangen. 57 Vgl. auch Risi 2017, S. 123; außerdem Le Roy 2007b.

$58 \mathrm{Vgl}$. Le Roy 2007a.

59 Risi 2017, S. 121. 
Richtungen reicht, wobei ein originäres Zentrum längst nicht mehr auszumachen ist beziehungsweise keine Rolle spielt. Andererseits verblüfft wie so oft in Stücken von Le Roy - die Art, wie der Choreograf einer solchen Komplexität begegnet: Mit betonter darstellerischer Einfachheit (er steht allein auf der Bühne und dirigiert) eröffnet er einen vielfältigen Reflexionsraum, der mehr Fragen aufwerfen als etwas Bestimmtes (wie etwa virtuosen Tanz im herkömmlichen Sinne) zeigen soll. In diesem Fall betrifft das Spektrum der angestoßenen Reflexion den Umgang mit Sacre als musikalisch-choreografischem Werk. Dieser beinhaltet auch dessen Vereinnahmung (etwa durch Royston Maldooms kommerziell und medial enorm erfolgreiches, Sozial'-Projekt) und reicht bis hin zum Verhältnis von Dirigieren und Tanzen / Choreografieren, ${ }^{60}$ womit auch die Macht der Geste im Kontext einer Orchesteraufführung thematisiert ist, ${ }^{61}$ oder auch generell in Bezug auf Wahrnehmung, Ästhetik und Funktion. Le Roys Sacre, so könnte man im Hinblick auf die in diesem Band verhandelte Debatte zusammenfassend sagen, referiert zwar ebenfalls auf ein Referenzsystem ohne ursprüngliches, Original'. Im Gegensatz aber zu Bausch, die diese Leerstelle in ihrer Fortschreibung innerhalb einer bestimmten Vorstellungswelt produktiv macht, umspielt Le Roys Reflexion die Sacre-Tradition gewissermaßen auf einer Metaebene. So geht er nicht auf Semantiken und Attribute des Sacre-Kanons ein, vielmehr interessiert er sich ,nur' für die komplexen Relationen und Bezüge als Verhältnisse, die sich befragen und weiterknüpfen lassen.

Ebenfalls als Ausgangspunkt für (eigene) Reflexionen fungiert die Vorstellungswelt von Le sacre du printemps in Frühlingsopfer des Performance-Kollektivs She She Pop. Das Stück wurde 2014 erstmals im Berliner HAU aufgeführt und tourte danach international, vor allem allerdings im Schauspiel- und Performance-, weniger im Tanzkontext. ${ }^{62}$ Dies hat vor allem einen Grund (neben Herkunft und Werdegang der Autor innen beziehungsweise des Kollektivs, das in den

60 Le Roy zit. nach Risi 2017, S. 124: "Looking at the mesmerizing performance of Simon Rattle conducting ,Le Sacre du Printemps', I felt he was dancing with the music as much as he was directing the orchestra".

61 Vgl. Risi 2017, S. 123, der wiederum auf Canettis Text Der Dirigent verweist.

62 Vgl. She She Pop 2014. 1990er Jahren aus dem Gießener Studiengang Angewandte Theaterwissenschaft hervorgegangen ist) ${ }^{63}$ : Basis des Stücks Frühlingsopfer von She She Pop bilden zwar wiederum Strawinskys Komposition sowie das kanonisch tradierte Wissen über Thematik, Aufbau und formale Aspekte des Bühnenwerks Sacre; allerdings wird in dessen 80 Minuten Dauer mehr gesprochen als zu Musik agiert. Sebastian Bark, einer der Performer, sagt zu Beginn, sie hätten „ein Ritual vorbereitet und das handelt davon, einander zu vereinnahmen, zu kontrollieren und dann abzustoßen und loszulassen. Dieses Ritual hat mehrere Teile. Der erste Teil heißt ,Vorrede', weil darin geredet wird. Später werden wir schweigen und Musik hören nämlich Strawinskys Frühlingsopfer. Diese Komposition hat zwei Teile. Aber vorher muss hier noch einiges ausgesprochen werden."64 Dieses Auszusprechende hat in charakteristisch postdramatischer She She Pop-Manier eine ebenso persönlich autobiografische wie verallgemeinerbar zeitkritische Dimension. ${ }^{65}$ Ihr Frühlingsopfer fokussiert auf das Opferthema, genauer auf "die Frage nach dem weiblichen Opfer in der Familie und in der Gesellschaft"; dabei setzen sich die Performer_innen mit ihren Müttern auseinander, die zwar nicht physisch, aber medial auf der Bühne präsent sind. ${ }^{66}$ Vier Mütter, drei Töchter und ein Sohn sprechen dabei in dramaturgisch (diskursiv wie optisch und räumlich) subtil arrangierten Szenen über ihr jeweiliges Verhältnis zu sich, untereinander, zu den Vätern und über soziale Rollenansprüche sowie Lebensentwürfe.

Den Teil, den Bark - nicht ohne Ironie - als "schweigen und Musik hören" und damit quasi als nonverbale ,Nebensache' im sonst gesprochenen Stück bezeichnet, bildet dann aber genau jenen Teil, der die Fortschreibung des Sacre im engeren Sinn ausmacht. Dabei ist aus dem Off Strawinskys Musik in voller Länge zu hören ${ }^{67}$

63 She She Pop (o. J.).

$64 \mathrm{Vgl}$. die vom Kollektiv der Autorin zur Verfügung gestellte Videoaufnahme einer Aufführung des Stücks in der Kaserne Basel vom Juni 2014, ab Min. 09:00.

$65 \mathrm{Vgl}$. dazu auch Matzke 2005.

66 Vgl. She She Pop (2014). Im Unterschied dazu agierten in Testament, dem bisher erfolgreichsten She She PopStück von 2010, die Väter der Performer_innen live mit auf der Bühne. Vgl. She She Pop (2010).

67 Nach Strawinskys integral gegebenem ersten Teil folgt in She She Pops Frühlingsopfer wieder eine gesprochene 


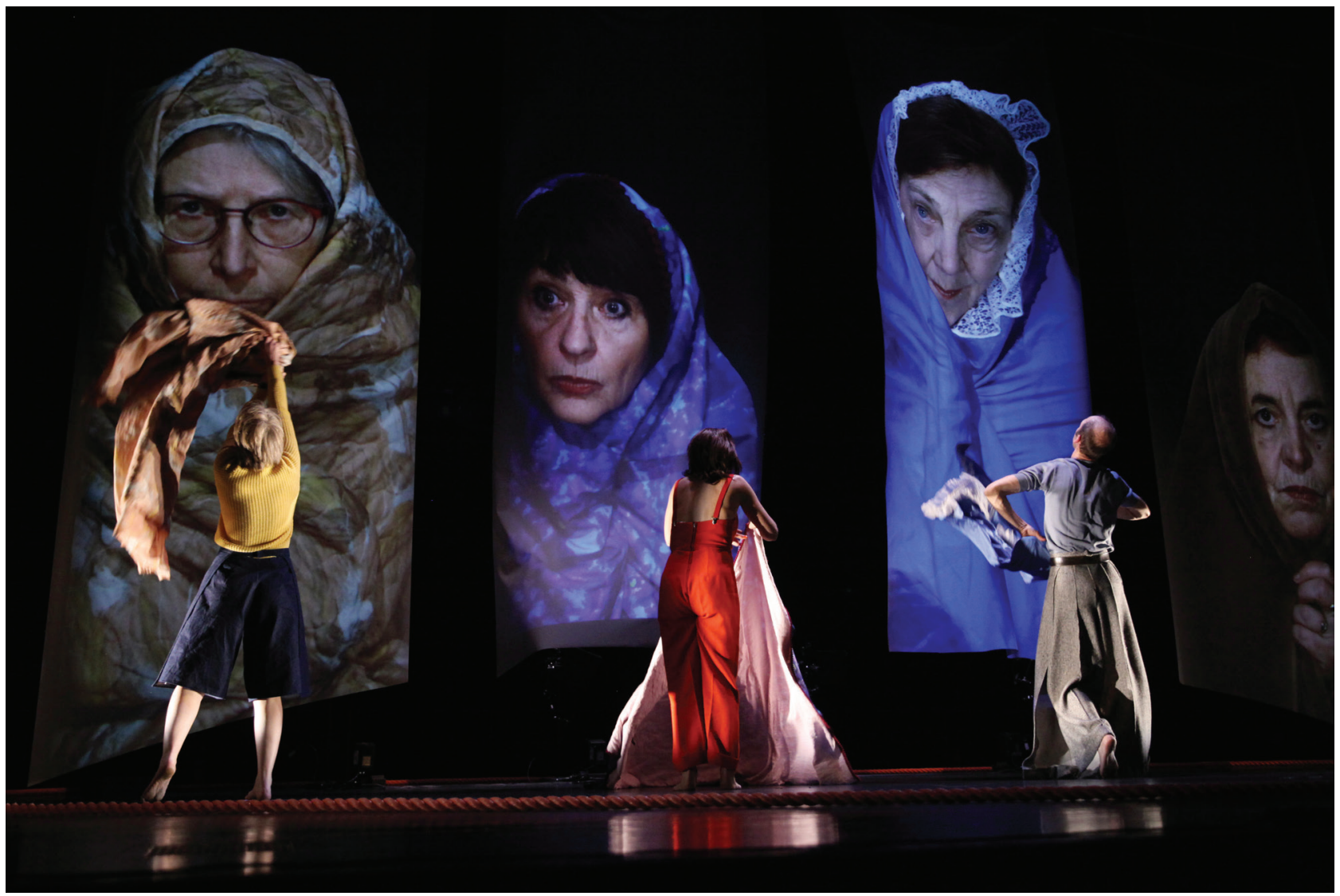

Abb. 2. She She Pop: Frühlingsopfer (2014), Bild: Doro Tuch

und die Performer_innen bewegen sich gemeinsam beziehungsweise abwechselnd oder in Überblendung mit den medial projizierten Müttern in einer genau festgelegten Choreografie. Ganz zum Schluss, nach dem Tanz, wird nochmals im Chor das soeben durchexerzierte Ritual kommentiert als zu veröffentlichender Ausdruck dessen, was "besonders war".68 Damit reflektiert dieses Stück in seiner wiederum eigenen Weise gerade auch den entscheidenden Widerspruch in der Fortschreibungsgeschichte von Sacre: Dessen absoluten bis transgressiven Charakter, auf den mit Garafola oben hingewiesen wurde, ${ }^{69}$ auf der einen Seite und auf der anderen das Pochen auf einer jeweiligen Besonderheit, einer immer wieder neu für sich in Anspruch genommenen ,Originalität', die sich von der ersten ,Original'-Fassung über die einzelnen - ganz unterschiedlich darauf Bezug nehmenden Versionen - hinzieht. Insofern bildet She She Pops Frühlingsopfer vorsätzlich

Passage vor dem zweiten Teil der Musik.

$68 \mathrm{Vgl}$. die Videoaufnahme (wie in Fn. 64) ab 1:16:35.

69 Garafola 2014, S. 179; vgl. Fn. 29. einen weiteren Sacre innerhalb von dessen netzwerkartiger Fortschreibungsgeschichte. Dieser reflektiert den Umstand der eigenen Einschreibung in einen Kanon genauso wie außerdem die künstlerischen, kulturellen, gesellschafts- und generationenspezifischen Kontexte, auf die dabei Bezug genommen wird. So sollte am Ende dieser Beispielreihe deutlich werden, dass man anhand der jüngeren Reproduktionshistorie von Sacre einerseits zeigen kann, wie die Bedingungen und Wirkungen von dessen künstlerischer Reproduzierbarkeit (mit-)reflektiert werden, und andererseits, wie spielerisch gerade auch mit Abweichungen umgegangen wird.

Schließlich bleibt bei einer solchen offenen, aber gleichzeitig immer wieder neu relational differenzierenden Betrachtungsweise zu konstatieren: Wovon genau wie abgewichen wird, bleibt meist (vorsätzlich) implizit und fußt in einer Vorstellungswelt, die jede_r selber in sich trägt, die gar nicht diffus sein muss, sondern sehr präzise sein kann, je nachdem, welchen Tanz er gesehen, wovon sie gehört oder gelesen hat. Das ,Original' gibt es nicht und gibt es doch - je in jeder 
Vorstellung, sei dies nun von Sacre oder von Schwanensee oder von einem anderen Tanzstück mit einer nicht-linearen, gleichwohl reichen Fortschreibungsgeschichte.

\section{Literaturverzeichnis}

Acocella, Joan / Greene, Jonnie / Garafola, Lynn (1992): The Rite of Spring Considered as Nineteenth-Century-Ballet. In: Ballet Review, 20/2, S. 68-71.

Archer, Kenneth / Hodson, Millicent (2000): Confronting Oblivion. Keynote Address and Lecture Demonstration on Reconstructing Ballets. In: Jordan, Stephanie (Hg.): Preservation Politics. Dance Revived, Reconstructed, Remade. Proceeding of the Conference at the University of Surrey Roehampton November 8-9, 1997. London: Dance Books, S. 1-20.

Baßler, Moritz / Schumacher, Eckhard (Hgg.) (2016): Handbuch Literatur \& Pop. Berlin: De Gruyter.

Bausch, Pina (1975): Das Frühlingsopfer. http://www. pina-bausch.de/de/stuecke/detail/show/das-fruehlingsopfer/ (17.8.2018).

Bausch, Pina (2012): Le sacre du printemps. Das Frühlingsopfer, The Rite of Spring. Tanztheater Wuppertal. Buch mit DVD. Paris: L'Arche.

Brandstetter, Gabriele (1995): Schwanensee. Zauber des Balletts. In: Schwanensee. Programmheft der Bayerischen Staatsoper München, März, S. 3-14.

Brandstetter, Gabriele (2014): Le sacre du printemps 1913 / 2013. In: Gygax, Raphael (Hg.): Sacré 101 - An Anthology on The Rite of Spring. Zürich: JRP / Ringier, S. $149-161$.

Brandstetter, Gabriele (2017): Tanz über Gräben. In: Brandstetter, Gabriele / Schneider, Katja (Hgg.): Sacre 1913 / 2013. Tanz, Opfer, Kultur. Freiburg i. Br.: Rombach, S. 17-48.

Brandstetter, Gabriele / Klein, Gabriele (Hgg.) (2007): Methoden der Tanzwissenschaft. Modellanalysen zu Pina Bauschs Le sacre du printemps. Bielefeld: transcript (= TanzScripte; 4).

Brandstetter, Gabriele / Klein, Gabriele (Hgg.) (2015): Methoden der Tanzwissenschaft. Modellanalysen zu Pina Bauschs Le sacre du printemps / Das Frühlingsopfer. 2., überarbeitete und erweiterte Neuauflage. Bielefeld: transcript (= TanzScripte; 32).

Brandstetter, Gabriele / Schneider, Katja (Hgg.) (2017): Sacre 1913 / 2013. Tanz, Opfer, Kultur. Freiburg i. Br.: Rombach.

Dahms, Sibylle (Hg.) (2001): Tanz. Stuttgart / Weimar / Kassel et al.: Metzler / Bärenreiter.

Danuser, Hermann / Zimmermann, Heidy (Hgg.) (2013): Avatar of Modernity. The Rite of Spring Reconsidered. London: Boosey \& Hawkes.

De Keersmaeker, Anne Teresa (2013-2014): Re:Rosas! http://www.rosasdanstrosas.be/en-home/ (9.8.2018).

Fanger, Iris M. (2004): Swan Lake. In: Cohen, Selma Jeanne (Hg.): International Encyclopedia of Dance,
Volume 6. New York / Oxford: Oxford University Press, S. 29-35.

Garafola, Lynn (2013): Diaghilev's Ballets Russes. A New Kind of Company. In: Danuser, Hermann / Zimmermann, Heidy (Hgg.): Avatar of Modernity. The Rite of Spring Reconsidered. London: Boosey \& Hawkes, S. 25-41.

Garafola, Lynn (2014): Ein Jahrhundert Le sacre du printemps. Die Etablierung einer Avantgarde-Tradition. In: Gygax, Raphael (Hg.): Sacré 101 - An Anthology on The Rite of Spring. Zürich: JRP / Ringier, S. 163-179.

Garafola, Lynn (2017): Ein Jahrhundert Le sacre du printemps. Die Etablierung einer Avantgarde-Tradition. In: Brandstetter, Gabriele / Schneider, Katja (Hgg.): Sacre 1913 / 2013. Tanz, Opfer, Kultur. Freiburg i. Br.: Rombach, S. 49-59.

Gygax, Raphael (Hg.) (2014): Sacré 101 - An Anthology on The Rite of Spring. Zürich: JRP / Ringier.

Hahn, Thomas (2013): Streit of Spring. In: Tanz, Aug./ Sept., S. 24-31.

Hartmann, Annette / Woitas, Monika (Hgg.) (2016): Das große Tanz Lexikon. Tanzkulturen, Epochen, Personen, Werke. Laaber: Laaber.

Hodson, Millicent (1980): The Fascination Continues. Searching for Nijinsky's Sacre. In: Dance Magazine, 54/6, S. 64-75.

Hodson, Millicent (1986 / 1987): Nijinsky's Choreographic Method. Visual Sources from Roerich for Le sacre $d u$ printemps. In: Dance Research Journal, 18/2, S. 7-15. Hodson, Millicent (1996): Nijinsky's Crime against Grace. Reconstruction Score of the Original Choreography for Le sacre du printemps. Stuyvesant, NY: Pendragon Press.

Hodson, Millicent / Archer, Kenneth (o. J.): Ballets Old \& New. http://www.hodsonarcher.com/ Hodson_Archer_-_Ballets_Old_\&_New/Le_Sacre_du_ Printemps.html (15.8.2018).

Hüster, Wiebke (2011): Beklaut Beyoncé Knowles Anne Teresa de Keersmaeker? In: Blog Aufforderung zum Tanz / Frankfurter Allgemeine Zeitung. https://blogs. faz.net/tanz/2011/10/10/beklaut-beyonce-knowles-anne-teresa-de-keersmaeker-70/ (24.5.2019).

Hutchinson Guest, Ann (2000): Is Authenticity to Be Had? In: Jordan, Stephanie ( $\mathrm{Hg}$.): Preservation Politics. Dance Revived, Reconstructed, Remade. London: Dance Books, S. 65-71.

Jäger, Ludwig (2019): ,Aura' und ,Widerhall'. Zwei Leben des ,Originals' - Anmerkungen zu Benjamins Konzeptionen des Originalen. In: Kulturwissenschaftliche Zeitschrift, 3, S. 9-22.

Jordan, Stephanie (Hg.) (2000): Preservation Politics. Dance Revived, Reconstructed, Remade. London: Dance Books.

Jordan, Stephanie (2007a): Machine Metaphors in Pina Bausch's The Rite of Spring. A Choreomusical Approach. In: Brandstetter, Gabriele / Klein, Gabriele (Hgg.): Methoden der Tanzwissenschaft. Modellanalysen zu Pina Bauschs Le sacre du printemps. Bielefeld: Transcript (= TanzScripte; 4), S. 159-169. 
Jordan, Stephanie (2007b): Stravinsky Dances. Re-Visions Across a Century. Alton: Dance Books.

Jordan, Stephanie (2013): Le sacre du printemps. Milestones in Dance. In: Danuser, Hermann / Zimmermann, Heidy (Hgg.): Avatar of Modernity. The Rite of Spring Reconsidered. London: Boosey \& Hawkes, S. 221-239.

Jordan, Stephanie (2017): The Rite of Spring as a Dance. Recent Re-visions. In: Neff, Severine / Carr, Maureen / Horlacher, Gretchen (Hgg.): The Rite of Spring at 100. Bloomington: Indiana University Press, S. 29-38.

Jordan, Stephanie / Nicholas, Larraine (2002): Stravinsky the Global Dancer. A Chronology of Choreography to the Music of Stravinsky. London: Roehampton University. http://urweb.roehampton.ac.uk/ stravinsky/ (14.8.2018).

Kessler, Harry Graf (2005): Das Tagebuch 1880-1937. In: Kamzelak, Roland S. / Ott, Ulrich / Schuster, Jörg (Hgg.): Das Tagebuch 1880-1937, Bd. 4. Stuttgart: Cotta.

Kieser, Klaus / Schneider, Katja (2002): Reclams Ballettführer. 13. Auflage. Stuttgart: Reclam.

Kraut, Anthea (2016): Choreographing Copyright. Race, Gender, and Intellectual Property Rights in American Dance. Oxford: Oxford University Press.

Le Roy, Xavier (2007a): Le sacre du printemps. Technical Rider. http://www.xavierleroy.com/page. php?ft $=$ a0f7e349ea6cabcd6b97cfb20f2582d9c062cba9\&lg=en (21.8.2018).

Le Roy, Xavier (2007b): Récit de travail sur Le sacre du printemps. In: Repères, cahier de danse, 20/2, S. 22-25.

Le Roy, Xavier (2018): Le sacre du printemps. http:// www.xavierleroy.com/page. $p h p ? s p=c a 9 f 423 d 10 e c-$ 10d5b62a0377dbe93e5f17b56487\&lg=en (17.8.2018).

Matzke, Annemarie (2005): Testen, Spielen, Tricksen, Scheitern. Formen szenischer Selbstinszenierung im zeitgenössischen Theater. Hildesheim: Olms.

Risi, Clemens (2017): Die performative Macht der Geste. Xavier Le Roy re-enactet Simon Rattle, der Strawinskys Le sacre du printemps dirigiert. In: Brandstetter, Gabriele / Schneider, Katja (Hgg.): Sacre 1913 / 2013. Tanz, Opfer, Kultur. Freiburg i. Br.: Rombach, S. 121-134.

Rivière, Jacques (2017): Le sacre du printemps. In: Brandstetter, Gabriele / Schneider, Katja (Hgg.): Sacre 1913 / 2013. Tanz, Opfer, Kultur. Freiburg i. Br.: Rombach, S. 305-326.

Schlicher, Susanne (1987): TanzTheater. Traditionen und Freiheiten. Pina Bausch, Gerhard Bohner, Reinhild Hoffmann, Johann Kresnik, Susanne Linke. Reinbek: Rowohlt.

Schneider, Katja (2016): Schwanensee (Lebedinoje osero). In: Hartmann, Annette / Woitas, Monika (Hgg.): Das große Tanz Lexikon. Tanzkulturen, Epochen, Personen, Werke. Laaber: Laaber, S. 550-552.
She She Pop (2010): Testament. https://sheshepop.de/ testament/ (21.8.2018).

She She Pop (2014): Frühlingsopfer. http://www. sheshepop.de/produktionen/fruehlingsopfer.html (21.8.2018).

She She Pop (o. J.): Alles über She She Pop. https:// sheshepop.de/ueber-uns/ (21.8.2018).

Thomas, Helen (2000): Reproducing the Dance. In Search of the Aura? In: Jordan, Stephanie (Hg.): Preservation Politics. Dance Revived, Reconstructed, Remade. London: Dance Books, S. 125-131.

Thomas, Helen (2004): Reconstruction and Dance as Embodied Textual Practice. In: Carter, Alexandra (Hg.): Rethinking Dance History. A Reader. London: Routledge, S. 32-45.

Thurner, Christina (2007): Prekäre physische Zone. Reflexionen zur Aufführungsanalyse von Pina Bauschs Le sacre du printemps. In: Brandstetter, Gabriele / Klein, Gabriele (Hgg.): Methoden der Tanzwissenschaft. Modellanalysen zu Pina Bauschs Le sacre du printemps. Bielefeld: transcript (= TanzScripte; 4), S. 47-58.

Thurner, Christina (2016): Rekonstruktion. In: Hartmann, Annette / Woitas, Monika (Hgg.): Das große Tanz Lexikon. Tanzkulturen, Epochen, Personen, Werke. Laaber: Laaber, S. 498-499.

Thurner, Christina / Wehren, Julia (Hgg.) (2010): Original und Revival. Geschichts-Schreibung im Tanz. Zürich: Chronos.

Wehren, Julia (2016): Körper als Archiv in Bewegung. Choreografie als historiografische Praxis. Bielefeld: transcript (= TanzScripte; 37).

Weickmann, Dorion (2018): Schwanensee. In: Tanz, Juli, S. $16-17$.

Winkler, Christoph (2012): Dance! Copy! Right? http://www.christoph-winkler.com/produktionen/ dancecopyright/ (15.8.2018).

Winkler, Christoph / Kieser, Klaus (2012): Kann es ein Urheberrecht an der menschlichen Bewegung geben? Interview. Tanz. http://www.tanzfonds.de/de/artikel/ interview-christoph-winkler (10.8.2018).

Woitas, Monika (2001): 20. Jahrhundert. In: Dahms, Sibylle (Hg.): Tanz. Stuttgart / Weimar / Kassel: Metzler / Bärenreiter, S. 136-152.

Zickgraf, Leila (2017): Igor' Stravinskijs Theater der Zukunft. Das Choreodrama Le sacre du printemps im Spiegel der ,Theaterreform um 1900'. Bisher unveröffentlichte Dissertation. Basel. 